\title{
Plasma Rotation Measurements by Passive Spectroscopic Method in the HYBTOK-II using a Dynamic Ergodic Divertor
}

\author{
Yu HASEBE, Masaaki OKAMOTO, Shin KAJITA ${ }^{1)}$, Noriyasu OHNO $^{2)}$ and Shuichi TAKAMURA ${ }^{3)}$ \\ Graduate School of Engineering, Nagoya University, Nagoya 464-8603, Japan \\ 1) Japan Atomic Energy Agency, Naka 311-0193, Japan \\ ${ }^{2)}$ EcoTopia Science Institute, Nagoya University, Nagoya 464-8603, Japan \\ ${ }^{3)}$ Department of Electronics, Aichi Institute of Technology, Toyota 470-0392, Japan
}

(Received 16 November 2007 / Accepted 6 February 2008)

\begin{abstract}
Plasma rotation or its shear is important in the formation of a transport barrier. It is believed that a rotating helical magnetic field generated by a dynamic ergodic divertor (DED) can generate a rotational torque in tokamak plasmas, and therefore control the rotation profiles. To measure the plasma rotations and investigate the effect of DEDs on them, we developed a passive spectroscopic measurement system for the small tokamak HYBTOK-II. A spontaneous toroidal plasma rotation in the co-current direction and poloidal plasma rotation in the electron diamagnetic drift direction have been observed without using a DED. Considerable changes in the plasma flow have been obtained using a DED for reducing toroidal and poloidal rotation velocities near the resonant magnetic surface. The change in the plasma rotation velocity was found to couple with the change in the radial electric field.

(c) 2008 The Japan Society of Plasma Science and Nuclear Fusion Research
\end{abstract}

Keywords: plasma rotation, dynamic ergodic divertor, passive spectroscopy, radial electric field, HYBTOK-II

DOI: $10.1585 / \mathrm{pfr} .3 . S 1015$

\section{Introduction}

For achieving high-performance magnetically confined plasmas, the control of plasma rotations and their profiles plays important roles in forming and sustaining a transport barrier by a modification of the radial electric field $[1,2]$. In burning plasmas, it is difficult to control plasma properties by external current drive or auxiliary heating (i.e., controlling the current and temperature profiles) due to large bootstrap current and self- $\alpha$ heating. The plasma rotation control can potentially control the plasma characteristics in the burning phase. In the present devices, neutral beam injection is used to control the plasma rotations as an external momentum source in studies of plasma properties.

The dynamic ergodic divertor (DED), which has been developed to control energy and/or particle transport at the edge region, is believed to generate a rotational torque in plasmas. A modulation of particle and/or energy transport in DED results from the magnetic field structure caused by magnetic islands induced by externally applying a rotating helical magnetic perturbation field (RHF) [3-7]. The mechanism of controlling plasma rotation using the DED relates to the Lorentz torque generated due to the interaction between the shielding current produced by the rotating perturbation field in plasmas and the perturbation field itself [7]. The torque is expected to be large when the relative angular frequency between the plasma and RHF in

author'se-mail: kajita@ees.nagoya-u.ac.jp the DED is large. However, the high-frequency RHF in the plasma becomes weak due to the shielding effect of the vacuum vessel, because the helical coils are wound outside of the vacuum vessel. On the other hand, the lower frequency RHF can penetrate through the chamber wall. Therefore, in this study, we focus on the lower frequency RHF ( several kHz) case for investigating the effect of stochastic magnetic field formed inside the separatrix.

To study the effect of the DED on plasma rotation in the small tokamak HYBTOK-II, an optical measurement system has been developed with the working gas of helium.

\section{Experimental Setup}

The experiments have been conducted on the small tokamak HYBTOK-II (the major radius $R_{0}=40 \mathrm{~cm}$, the plasma minor radius $a \approx 10 \mathrm{~cm}$, the limiter radius $a_{\mathrm{w}}=$ $11 \mathrm{~cm}$ ). In the machine operation, the main discharge parameters were as follows: the plasma current was $I_{\mathrm{p}}=$ $5 \mathrm{kA}$, the toroidal magnetic field was $B_{\mathrm{t}}=0.28 \mathrm{~T}$, and the pulse duration time was 10 milliseconds. The HYBTOK-II is equipped with DED coils that comprise two sets of coils wound on the out-vessel locally in the toroidal direction. The RHF with the poloidal and toroidal mode numbers of $m / n=6 / 1$ resonates with the magnetic rational transform at $q=6$ magnetic surface (see Fig. 1) [5]. These two sets of coils are powered independently using an insulated gate bipolar transistor inverter power supply with a phase difference of $\pm 90^{\circ}$. We can control the poloidal rotation di- 
(a)

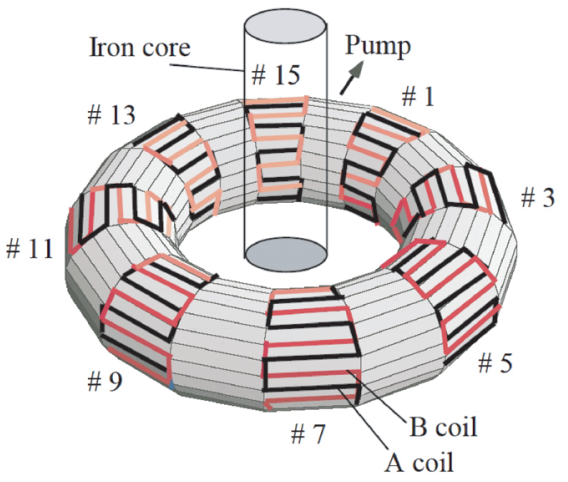

(b)

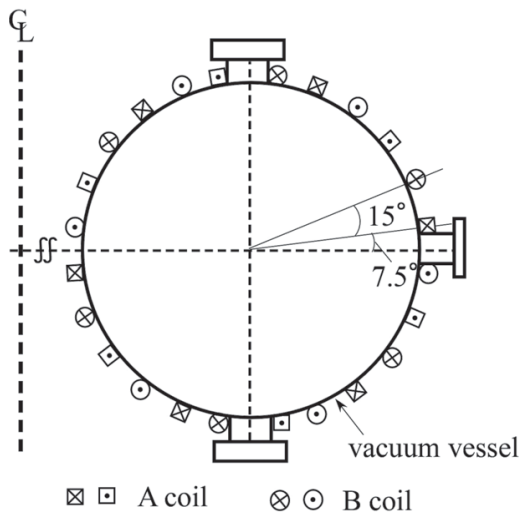

Fig. 1 Arrangement of the DED coils: (a) bird's eye view, (b) on poloidal cross sectional one [5].

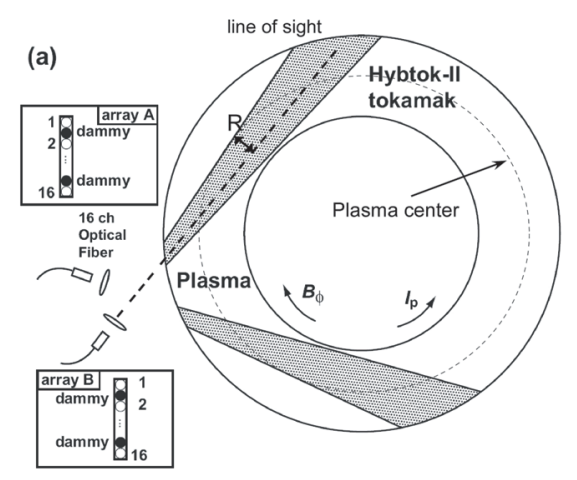

(b)

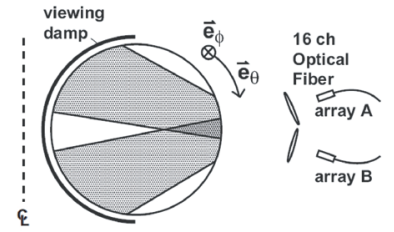

(c)

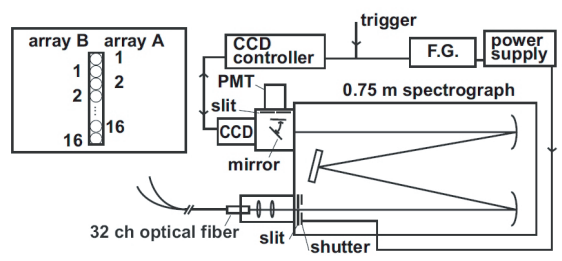

Fig. 2 Optical arrangements for (a) toroidal and (b) poloidal flow velocity measurements. (c) Spectrometer, detection device and synchronized system with external trigger.

rection of RHF by changing the phase difference between the two coils. The rotation frequency of RHF was selected as $3 \mathrm{kHz}$. Duration of the RHF was $10 \mathrm{~ms}$.

The optical measurement system consists of bifurcated 32-channel $(16 \mathrm{ch} \times 2)$ optical fiber arrays, a focusing lens (103-mm focal length for the toroidal direction, 34.5-mm for the poloidal direction), a Czerny-Turner spectrometer (1800 grooves/mm grating, 1.0-m focal length and $0.01-\mathrm{nm}$ resolution), and a $1024 \times 256$-pixels charge coupled device (CCD) detector (see Fig. 2). By changing the position of the fiber arrays using movable stages, the distance between the line of sight and the plasma center in the equatorial plane can be varied from 0 to $100 \mathrm{~mm}$. The flow velocity $V$ is obtained by $V=c \cdot \Delta \lambda / \lambda$, where $c$ is the speed of light, $\Delta \lambda$ is the Doppler shift, and $\lambda$ is the central wavelength of the observed spectrum. The shifted value $\Delta \lambda$ was estimated using $\Delta \lambda=\left(\lambda_{\text {blue }}-\lambda_{\text {red }}\right) / 2$ for this system, where $\lambda_{\text {blue }}$ and $\lambda_{\text {red }}$ are the central wavelength of the spectrum observed from the upstream and downstream of the plasma flow, respectively. Therefore, one branch of the bifurcated arrays faced the upstream side and another faced towards the downstream side, as shown in Fig. 2. In the experiments, singly ionized helium emission He II ( $n=3-4$, $\lambda=468.54-468.59 \mathrm{~nm}$ ) was employed for flow measurements. The spectrum was fitted with multi-Gaussian functions considering a fine structure of the associated transitions.

A triple probe, which is accessible to the center of the plasma column, is installed for the measurement of the electron temperature $T_{\mathrm{e}}$, electron density $n_{\mathrm{e}}$, and floating potential $V_{\mathrm{f}}$. The plasma potential $V_{\mathrm{p}}$ is estimated using the relation $V_{\mathrm{p}}=V_{\mathrm{f}}+3 T_{\mathrm{e}}$.

\section{Results and Discussion \\ 3.1 Rotation velocity measurements}

In the measurements, pure helium gas was employed as the working gas to improve the $\mathrm{S} / \mathrm{N}$ ratio by obtaining a strong He II emission. Typical electron density and electron temperature are $n_{\mathrm{e}} \sim 6 \times 10^{18} \mathrm{~m}^{-3}$ and $T_{\mathrm{e}} \sim 30 \mathrm{eV}$, respectively. First, we briefly present the fundamental spectral measurements obtained from the passive spectroscopy. Figure 3 shows the spectra observed both at the upstream and downstream sides with the relative strength of the finestructure multiplet. The observed spectra are well fitted by multi-Gaussian functions. The ion temperature obtained 


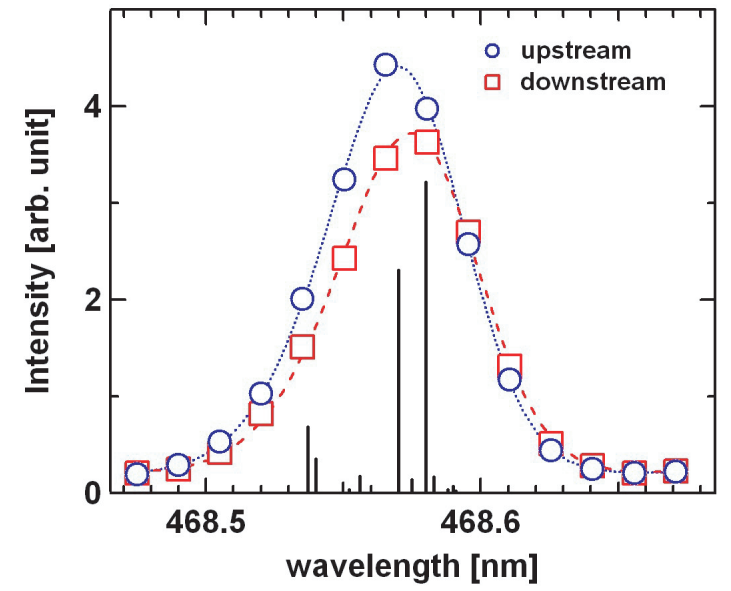

Fig. 3 Observed spectra at both upstream- (open circle) and downstream-side (open square), with fitted curves.

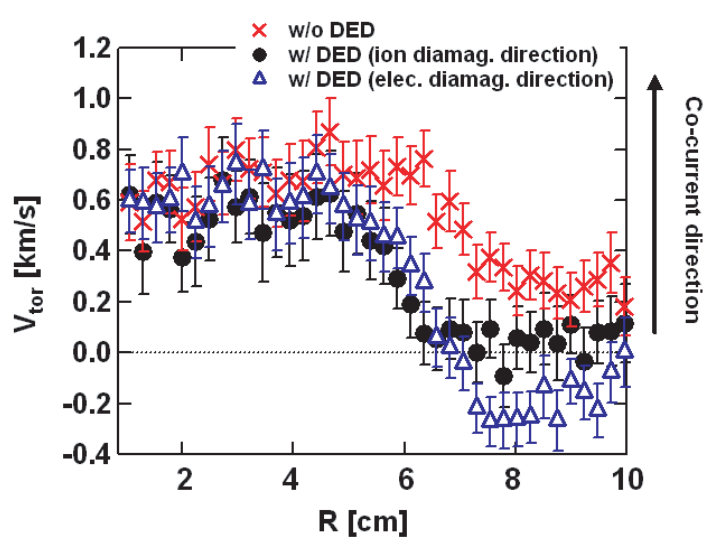

Fig. 4 Toroidal rotation velocity profile without (cross) and with DED in the direction of ion diamagnetic drift (closed circle) and in that of electron (triangle).

from the Doppler width, which is determined by considering the instrumental width, and the fine structure is about $5 \mathrm{eV}$, and it is found that there is no clear radial positional dependence for the ion temperature. The toroidal plasma rotation velocity profiles are shown in Fig. 4 as a function of the distance $R$ between the line of sight and the center of the plasma column in the equatorial plane, which is $1 \mathrm{~cm}$ inside from the center of the vacuum vessel, as shown in Fig. 2 (a). The errors of the rotation velocity in the graph come from the fitting errors. It is noted that the velocity does not show the local value because of the limitation of passive spectroscopy owing to line integration. Therefore, only the averaged value over the line of sight is currently available, particularly for the toroidal direction. A spontaneous plasma rotation in the co-current direction has been observed. The maximum velocity is about $0.8 \mathrm{~km} / \mathrm{s}$. By applying the maximum coil current of $150 \mathrm{~A}$ to the RHF, a reduction of the plasma rotation is clearly observed around $R=6 \mathrm{~cm}$ in the both cases of DED, i.e., in the direction

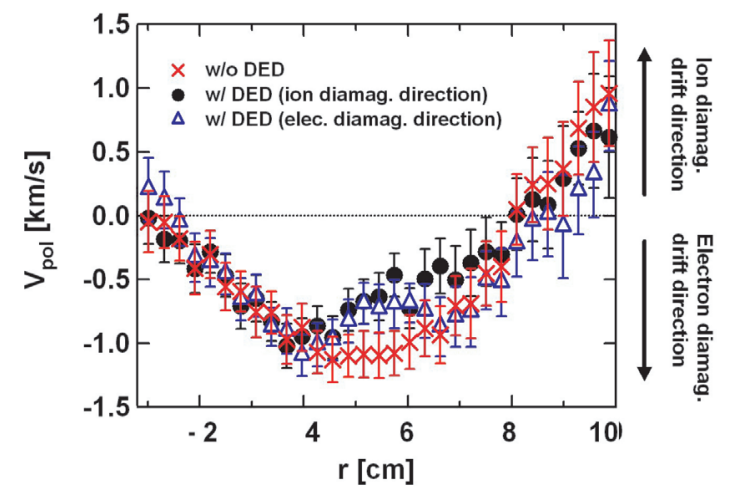

Fig. 5 Poloidal rotation velocity profile. The markers of each case are the same as those in Fig. 4.

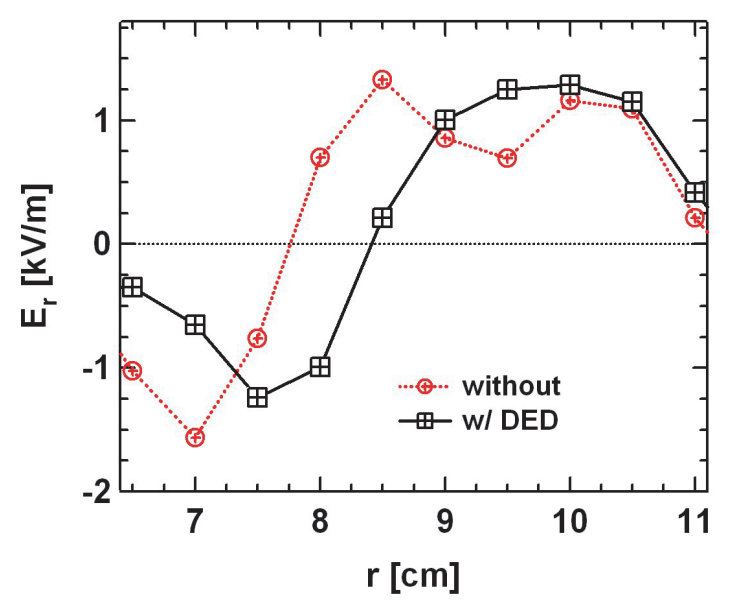

Fig. 6 Radial electric field evaluated by using the triple probe. Negative $E_{r}$ means inward field.

of the ion diamagnetic drift (denoted by closed circle) and in the direction of the electron diamagnetic drift (denoted by open triangle). Figure 5 shows the poloidal plasma rotation velocities without and with the $3 \mathrm{kHz}$ DED. " $r$ " in horizontal axis indicates the distance between the line of sight and the plasma center in the poloidal cross section. Without the DED, the direction of the plasma rotation is the same as that of the electron diamagnetic drift from $r$ $=2$ to $r=8 \mathrm{~cm}$. In the case in which the DED is used, the plasma rotation is damped around $r=6 \mathrm{~cm}$. The radial electric field $E_{r}$ derived using the plasma potential profile is shown in Fig. 6 for both without and with DED cases. The direction of $E_{r} \times B$ drift matches that of the poloidal rotation, and a reduction of $E_{r}$ at inside $r \sim 7.5 \mathrm{~cm}$ agrees with the reduction of the poloidal rotation. On the other hand, at the region from $r \sim 7.5$ to $9 \mathrm{~cm}$ where the last closed flux surface has lain, the difference in the values of $E_{r}$ between the without and with DED cases was positive, and the inversion point of $E_{r}$ was shifted outward. In this region the poloidal rotation was not affected by the DED, but the toroidal rotation reduced. As suggested by the force 
balance for ion fluid described Sec. 3.2, the toroidal rotation caused the change in the electric field. The variations in the plasma rotations using the DED are found to be coupled with radial electric field.

\subsection{Magnetic structure and electric field}

In the low driving frequency case $(3 \mathrm{kHz}$ DED), the change in the toroidal rotation due to the DED was observed at the location, where the poloidal rotation changed using the DED. The perturbation component of the radial magnetic field $B_{r 1}$ was measured using a radially movable magnetic probe, as shown in Fig. 7 (a). Figure 7 (b) shows the radial profile of the measured safety factor. $B_{r 1}$ in vacuum decreases as $r^{5}$, along the lines going into the chamber according to the multipolar $r^{m-1}$ approximation, where $m$ is poloidal mode number. However, $B_{r 1}$ in the plasma was amplified compared with that in vacuum near $q=6$ resonance surface $(r \approx 7.6 \mathrm{~cm})$, which mainly resonates to RHF. This is attributed to a re-distribution of plasma current due to the growth of a series of the magnetic islands [6]. Figure 8 (a) shows the calculated Poincaré mapping of magnetic field lines passing through a poloidal cross section, considering the RHF. The plasma current profile is assumed to be a parabolic distribution. The total plasma current is equal to that in the experiments; however, the

(a)

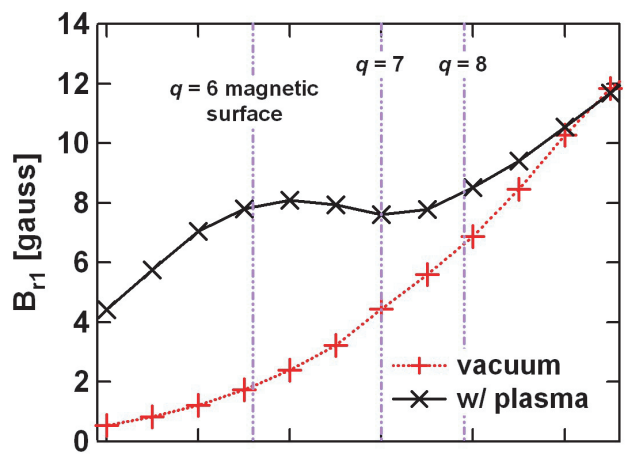

(b)

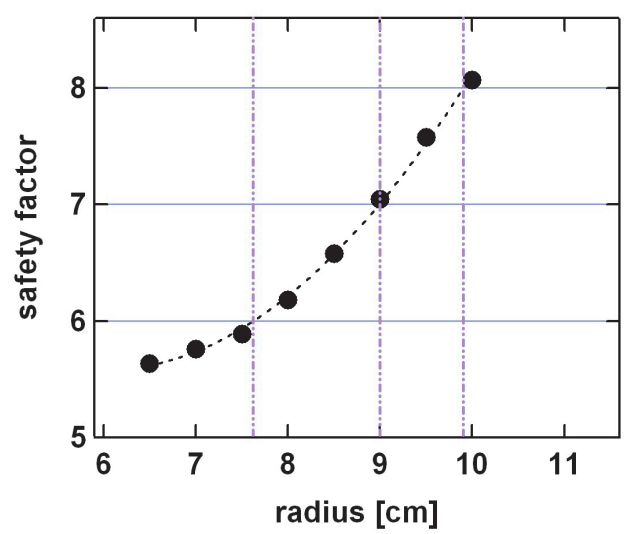

Fig. 7 (a) Perturbation component of radial magnetic field. The locations of rational surface of $q=6,7,8$ are shown by vertical lines, (b) measured safety factor profile. calculated $q$ profile in Fig. 8 (b) is slightly different from that in Fig. 7 (b). In fact, Fig. 8 clearly indicates the existence of magnetic islands, not only those of the $6 / 1$ mode but also of the 5/1 mode, which is the sideband component in this DED configuration. Ergodization of the magnetic structure occurs due to islands overlapping outside $q=6$ resonance surface. Considering the arguments on the edge magnetic structure, it is believed that the poloidal rotation damping was caused by the enhancement of poloidal parallel viscosity due to the island formation. In steady-state plasmas, the radial component of equation of motion of an ion fluid in the toroidal coordinates is given as follows:

$$
E_{r}=\left(1 / e Z_{\mathrm{i}} n_{\mathrm{i}}\right)\left(\partial P_{\mathrm{i}} / \partial r\right)-V_{\theta} B_{\phi}+V_{\phi} B_{\theta} .
$$

Here, $e Z_{\mathrm{i}}, n_{\mathrm{i}}$, and $P_{\mathrm{i}}$ are the electric charge, number density, and pressure of the ion component, respectively. $V$ and $B$ are the flow velocity and magnetic field, respectively. The changes in $E_{r}$ estimated from Eq. (1) using the results of the rotation measurements are in agreement with the experimental results. On the other hand, the decrease in the toroidal rotation seems to be caused by a friction increase

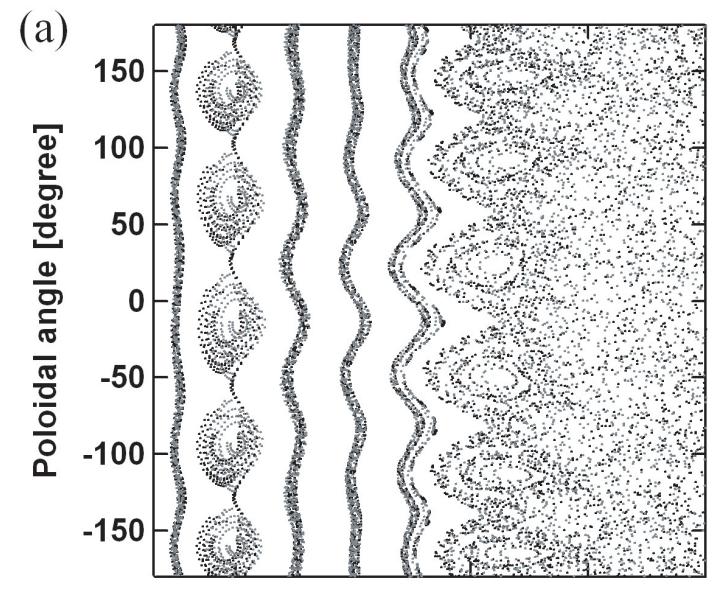

(b)

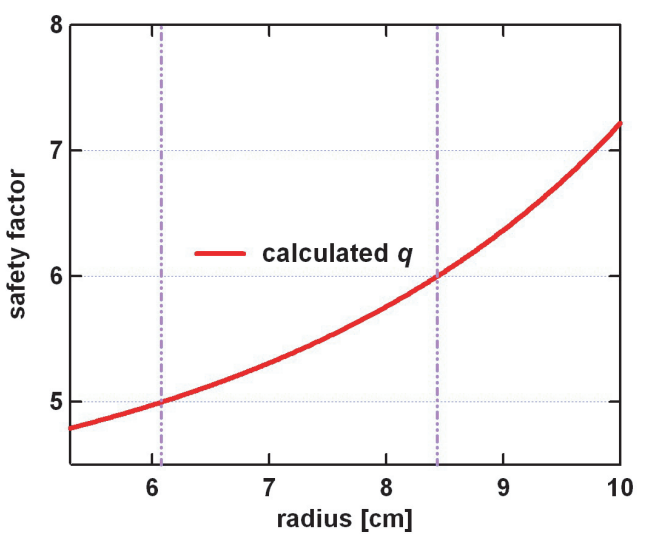

Fig. 8 (a) Poincaré plot of field lines near resonance surface. There are magnetic islands at $q=5$ (sideband mode), 6 (fundamental mode) rational surfaces. (b) Calculated $q$ profile. 
due to variation in the magnetic structure at the edge region, or friction with neutral helium that exists nearer to the edge region. Therefore, it could be said that the changes of the rotations originate in the magnetic island structure, and consequently the $E_{r}$ changes in these cases. However, it should be noted that an electrical short-circuit between the plasma edge and the wall, caused due to a contact of the field lines and the wall attributable to edge stochastic field structure, could also modify the radial electric field [3].

\section{Conclusion}

The changes in plasma rotation properties using a DED were reported. The plasma flow was measured by the optical emission spectroscopy developed in HYBTOKII. Doppler shift of the He II line obtained from the system is sufficiently large to estimate the rotation velocity and its direction in a pure helium discharge. The spontaneous toroidal plasma rotation in the co-current direction and the poloidal rotation in the electron diamagnetic drift direction were observed without using a DED. By applying a low-frequency RHF ( $\sim 3 \mathrm{kHz})$, both the toroidal and the poloidal rotations decreased. It is likely that they are caused by magnetic island formation. A change in radial electric field was also observed.

In the future, it is necessary to generate plasmas with higher electron temperature to study influences of shielding current in these plasmas. As modifications of radial electric field due to enhancements of electron losses in the stochastic region are observed on the TEXTOR $[10,11]$, there is also a need to consider these effects.

\section{Acknowledgments}

The authors thank Dr. Y. Kikuchi for mapping calculations and Mr. S. Hosoi for plasma feedback control system and DED operation.

[1] K. Ida, Phys. Fluids B 4, 2552 (1992).

[2] Y. Sakamoto et al., Nucl. Fusion 41, 865 (2001).

[3] S. Takamura, H. Yamada and T. Okuda, Nucl. Fusion 28, 183 (1988).

[4] M. Kobayashi, H. Kojima, K. Zhai and S. Takamura, Phys. Plasmas 7, 3288 (2000).

[5] S. Takamura, Y. Kikuchi, Y. Uesugi and M. Kobayashi, Nucl. Fusion 43, 393 (2003).

[6] M. Kobayashi et al., Nucl. Fusion 40, 181 (2000).

[7] Y. Kikuchi et al., J. Nucl. Mater. 313-316, 1272 (2003).

[8] Y. Kikuchi et al., Plasma Phys. Control. Fusion 49, A135 (2007).

[9] S. Kado and T. Shikama, J. Plasma Fusion Res. 79, 841 (2001).

[10] K. H. Finken et al., Phys. Rev. Lett. 94, 015003 (2005).

[11] B. Unterberg et al., J. Nucl. Mater. 363-365, 698 (2007). 